\title{
Forming Analysis of Bulge Formed Joints Based on Adaptive Finite Element Method
}

\author{
Jinjin ZHAI*, Yuantao SUN**, Qing ZHANG***, Xianrong QIN**** \\ *School of Mechanical Engineering, Tongji University, 200092 Shanghai, China, E-mail: zhaijinjin5200@sina.com \\ **School of Mechanical Engineering, Tongji University, 200092 Shanghai, China, E-mail: sun1979@sina.com \\ ***School of Mechanical Engineering, Tongji University, 200092 Shanghai, China, E-mail: zhqing_tj@126.com \\ ****School of Mechanical Engineering, Tongji University, 200092 Shanghai, China, E-mail: tjqin@tongji.edu.cn \\ crossref http://dx.doi.org/10.5755/j02.mech.24263
}

\section{Introduction}

Bulge formed joint is a new type of joint applied in truss structure, as shown in Fig. 1. It can reduce the stress concentration and improve the ultimate bearing capacity of truss structures. Forging forming is used to manufacture the joint and the cost is less than traditional joint [1]. But in the production process, we need to pay attention to the failure problems, such as the wear of the die, the breakdown of the workpiece, springback and so on. The Finite Element Method (FEM) is used to analyze the forming process of bulge formed joint.

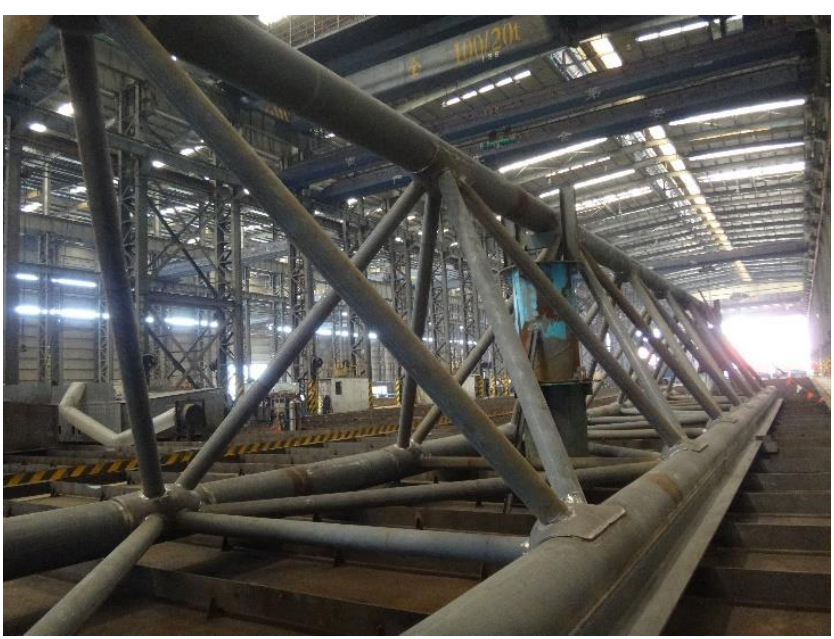

a

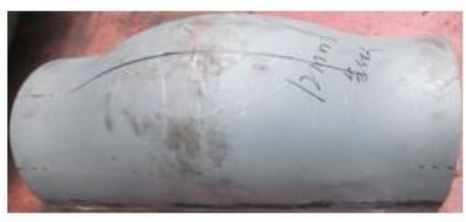

b

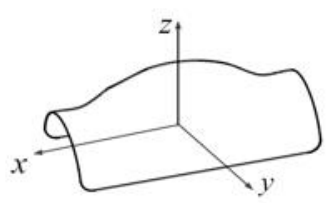

c
Fig. 1 The sketch map of bulge formed joint

Currently, the finite element method has been widely used in some fields. Marandia et al. studied the temperature rate coupled fracture problem of ductile materials using the finite element method, and solved it in ABAQUS commercial finite element program through user element subroutine (UEL) [2]. Seventekidis et al. proposed a combination method of optimal finite element and dynamic finite element and applied it to structural health monitoring (SHM)[3]. Xia Feng et al. simulated the forming process of
6063 aluminium alloy with finite element method in DEFORM-3D and studied the simulation method, the influences of different extrusion parameters, such as extrusion temperature, extrusion speed and extrusion ratio, on the extrusion process [4].

However, when solving nonlinear problems involving large deformation such as metal bulk forming problem, the grids may be seriously distorted. When the distortion accumulates to a certain extent, the calculation accuracy will be reduced, and even the calculation cannot continue. Chen et al. pointed out that the finite element method in landslide simulation has the shortcomings of the lack of mesh discretization objectivity and the difficulty of mesh adjustment [5]. Li pointed out that when using the finite element method to analyse the large deformation problems such as sheet metal stamping, collision and so on, due to the large amount of local element deformation, the grid is prone to distortion, which seriously affects the calculation accuracy. When the grid's distortion reaches a certain degree, even causes non-convergence, resulting in calculation interruption [6]. Although the finite element model can be reconstructed, on the one hand, the transformation between new and old grids will bring new errors; on the other hand, the reconstructed grids increase the workload and reduce the efficiency.

In view of the inherent defects of the finite element method, the finite element method has been improved. Khaji and Zakian combined stochastic finite element with spectral finite element as a new numerical tool to quantify uncertainty and applied it to engineering system reliability analysis [7]. Javanmardi and Maheri used the extended finite element method to predict the crack initiation and propagation direction of 3D solid concrete [8]. However, those advanced finite element methods, such as the extended finite element method and submodel method, are suitable for crack growth analysis, while the new meshless method has the disadvantages of long calculation time and low efficiency [9]. For metal forming, adaptive finite element method is more mature. The adaptive finite element method improves the calculation algorithm automatically according to the error of the calculation results, such as h-type, p-type and r-type adaptive finite element method [10]. Locally grids refinements are used in h-type adaptive finite element method. The initial grids can be roughly divided, and the error can be analyzed according to the analysis results. Then the grids can be refined by choosing the nodes with large error, and the process can be repeated many times until the accuracy requirement is satisfied. In this way, the grids reconstruction can be avoided, and the computational load can be reduced, 
and the accuracy of the analysis can be guaranteed.

The forming process of bulge formed joint is a typical nonlinear large deformation process. The error caused by grids distortion in the finite element analysis must be faced. Therefore, the adaptive finite element method proposed in this paper is used to analyze the forming process. The comparison of analysis results and experiment results shows that the adaptive finite element method is effective and the computational efficiency is improved.

In this paper, in Section 2 the basic principle of AFEM is introduced, including error calculation model and adaptive grids refinement strategy. In Section 3 the finite element model of bulge formed joint for forming process analysis is established. In Section 4 is the adaptive analysis of forming process of bulge formed joint and the comparison with the experimental results, and in Section 5 is the conclusion.

\section{The basic principle of Adaptive FEM}

There are many kinds of AFEM, including h-type, p-type, r-type and hp-type and so on. Among them, h-type AFEM mainly relies on grid refinement, p-type mainly relies on changing the location of nodes, and r-type mainly relies on the order of shape function to realize the adaptive analysis process. In view of the fact that the metal plastic forming process is a non-linear large deformation problem, which will lead to grid distortion, in this paper h-type AFEM is adopted.

Based on error estimation, h-adaptive finite element method refines the element grids whose error exceeds the preset range, and then the model is recalculated. Repeat the process until the target accuracy is achieved. H-type AFEM has the advantages of strong adaptability and good portability, but it requires higher error calculation model.

The h-type AFEM is used in this paper. Where, specific ideas as follows:

1. The finite element model is established and the grids are roughly divided;

2. According to the results, the nominal strain energy of each node is calculated and the standard deviation of nominal strain energy of each node is obtained;

3. If the error is less than the given accuracy, the calculation shall be finished and the result shall be output. Otherwise, the area with large error is determined and the grids are refined based on dichotomy, and then go back to the second step.

Among them, there are two main key technology:

1. an error estimation method is designed to calculate the error distribution.

2. Use grid refinement strategy to acquire more accurate model.

\subsection{The error estimation model}

In this paper, a new posterior error estimation method is used. Its index is the standard deviation of nominal strain energy of nodes. The specific ideas are as follows.

The governing equations of plasticity problems defined on boundary $\Gamma$ and domain $\Omega$ is:

Equilibrium equation:

$$
\sigma_{i j, j}=0
$$

Geometric equations:

$$
\dot{\varepsilon}_{i j}=\left(u_{i, j}+u_{j, i}\right) / 2 \text {, }
$$

where: $\dot{\varepsilon}_{i j}$ is strain rate tensor; $u$ is velocity field vector.

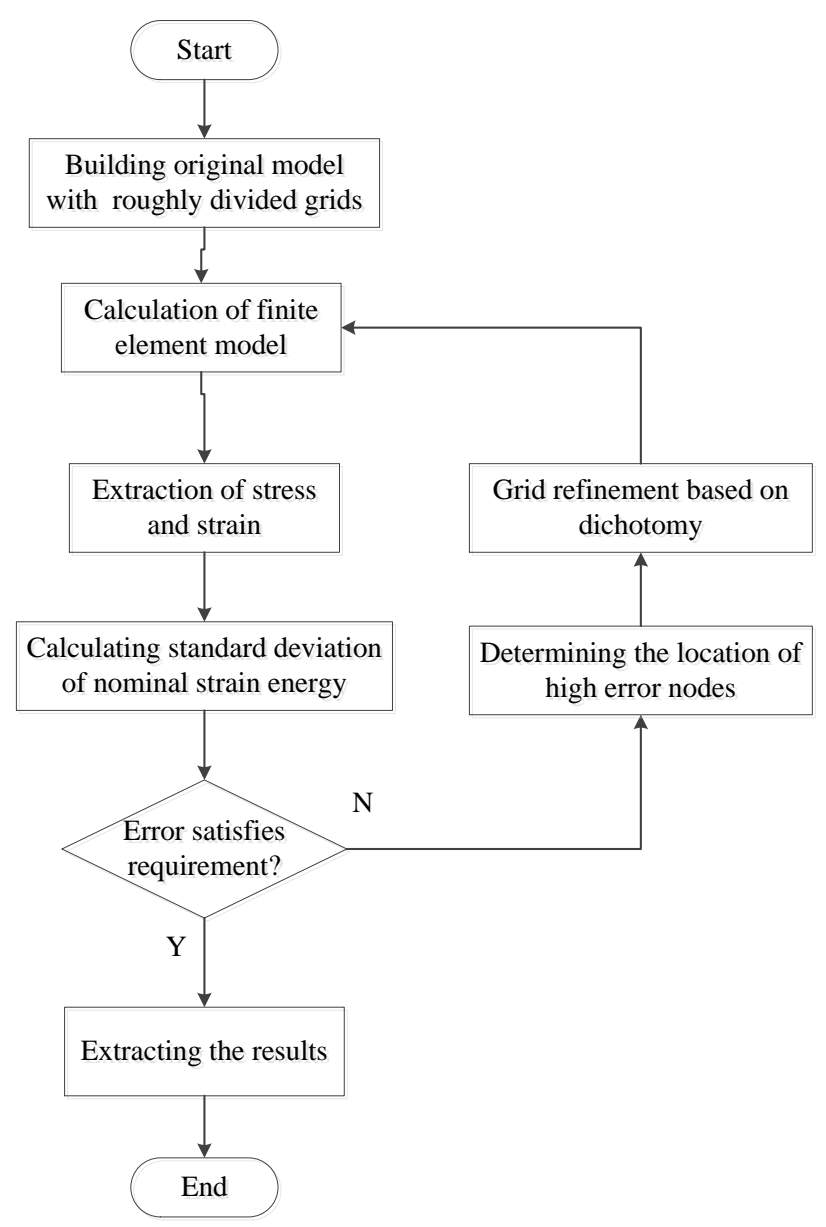

Fig. 2 Block diagram

Boundary conditions:

$$
\begin{aligned}
& \sigma_{i j} n_{j}=\bar{F}_{i} \quad\left(\text { in } \Gamma_{t}\right), \\
& u_{i}=\bar{u}_{i} \quad\left(\text { in } \Gamma_{u}\right),
\end{aligned}
$$

where: $\sigma_{i j}$ is stress tensor; $\varepsilon_{i j}$ is strain tensor; $u_{i}$ is displacement; $\Gamma_{t}$ and $\Gamma_{u}$ are the stress boundary and displacement boundary of region, the boundary region of $\Omega$ is $\Gamma, \Gamma=\Gamma_{t} \cup \Gamma_{u}: n_{j}$ is the outer normal line direction cosine of boundary $\Gamma ; \bar{u}_{i}$ is the given displacement on $\Gamma_{u}$.

The error caused by grids comes from discretization error and numerical integral error [11]. The discretization error of the model is due to the finite number of functions in the polynomial basis of shape function. Therefore, without changing the order of shape function, the error can only be reduced by mesh refinement.

Although the precision of initial calculation model with sparsely grids is low, it provides the basis for the variation of strain field and displacement field. Firstly, the node strain energy $W$ can be calculated by using stress and strain. The formula is as follows: 


$$
W=\frac{1}{2} \overline{\sigma \varepsilon},
$$

where: $\bar{\sigma}$ is the equivalent stress vector; $\overline{\boldsymbol{\varepsilon}}$ is the equivalent strain vector.

After meshing, each cell contains 6 or 8 nodes, as shown in Fig. 3. After the analysis, the nominal strain energy of each node can be obtained by further calculation. The nominal strain energy gradient is defined as follows:

$$
G_{i}^{W}=\frac{W}{\Delta x_{i}} \quad i=1,2,3 .
$$

$G_{i}(i=1,2,3)$ represents nominal strain energy gradients in the $x, y$ and $z$ directions, respectively. $\Delta x_{i}$ is the length of the element in the $x, y$ and $z$ directions.

It can be seen from Eq. (6) that in the region with large gradient, the nominal strain energy values of two adjacent nodes are quite different. Therefore, the calculation of the nominal strain energy gradient can be transformed into the statistical data of the nominal strain energy of each node of the same element, that is, the standard deviation of the nominal strain energy of each element. If the standard deviation is large, it means that the nominal strain energy of the nodes included in the element has a large difference, that is, the gradient is large. The standard deviation of nominal strain energy of each unit is calculated as follows:

$$
s=\sqrt{\frac{1}{n} \sum_{i=1}^{n}\left(W_{i}-\bar{W}\right)^{2}},
$$

where: $s$ is the standard deviation of nominal strain energy; $W_{i}$ is the nominal strain energy of this node in the same element; $\bar{W}$ is the average value of nominal strain energy; and $n$ is the number of the node in the element.

Given the initial criterion $s_{0}$, when $s>s_{0}$, it shows that the error is large, and the grid needs to be refined to obtain more accurate analysis results.

\subsection{Grid refinement strategy}

Based on the error calculation model, the location of the grid that needs to be refined is determined. After marking the corresponding elements, the grid refinement is required according to certain strategies.

In this paper, the principle of dichotomy is used to refine the grid. The main idea is to use the vertices of the

a) The actual forging process

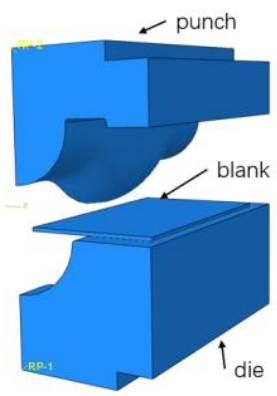

b) The geometric model marked element to insert new vertices on each edge of the element, so as to refine the target mesh. Two-dimensional and three-dimensional cases are shown in Figs. 3, a and b.

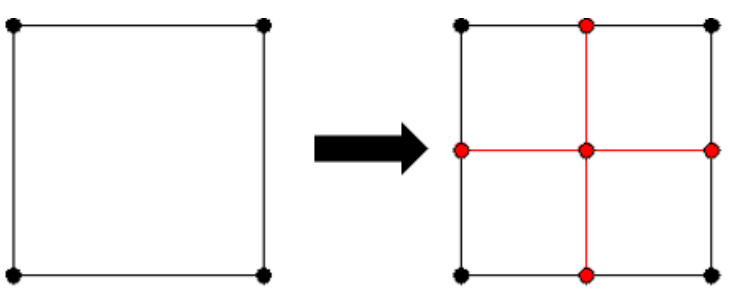

a) The two-dimensional case

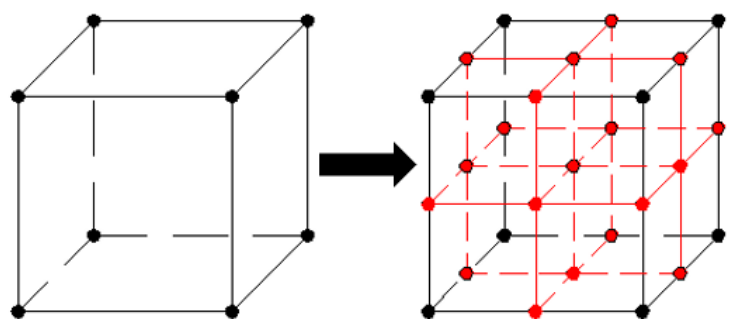

b) The three-dimensional case

Fig. 3 The sketch map of grid refinement

\section{Building of the Finite Element Model}

\subsection{FE model of Bulge Formed Joint}

In this paper, finite element software Abaqus/Explicit is used to analyze the forging process of bulge formed joints. Because of the geometric symmetry of forging forming of bulge formed joints, only $1 / 2$ of the complete structure of forging finite element model is taken to reduce the analysis time. Firstly, according to the actual production drawings shown in Fig. 4a, the three-dimensional models of the punch, the die and the sheet metal blank involved in the bulge formed joint forming process are established by using the software SolidWorks, and then the three-dimensional model is imported into the finite element software ABAQUS by using the X_T format, as shown in Fig. 4b. In the finite element model, the length of punch and die are $1100 \mathrm{~mm}$, width $500 \mathrm{~mm}$, height $618.8 \mathrm{~mm}$ and $502 \mathrm{~mm}$ respectively. Temperature-displacement coupled 3D4T solid elements are used in the punch and die, and 177930 and 39322 tetrahedral elements are obtained by Free method respectively. Temperature-displacement coupled S4RT shell element is used for sheet metal, and the thickness integral point is 5 . 450 hexahedral elements are divided by Structured method. The final meshing is shown in Fig. 4, c.

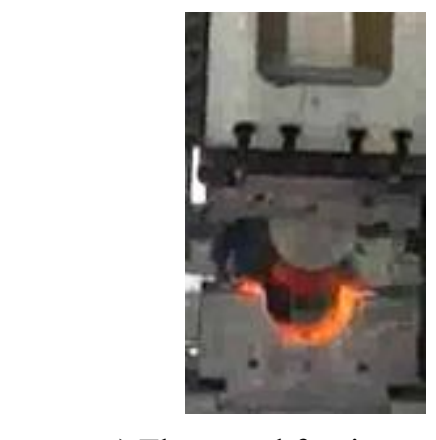

Fig. 4 The model of bulge formed joint 


\subsection{Constitutive model of Q345 steel}

Because the forming of inclusive joints is a hot forging process, it is necessary to fully consider the influence of temperature and strain rate on the properties of sheet metal materials, so as to establish an accurate finite element model to simulate the actual situation. Therefore, the Q345 constitutive model adopted in this paper refers to the revised Johnson-Cook model in reference [12]. The model synthetically describes the work hardening effect, strain rate effect and temperature softening effect of metal materials. Its expressions are as follows:

$$
\left\{\begin{array}{c}
\sigma_{y}=\left(A+B \varepsilon_{p}^{n}\right)\left(1+C \ln \frac{\dot{\varepsilon}}{\dot{\varepsilon}_{0}}\right)\left\{1-\left[\frac{T-<\frac{\dot{\varepsilon}-1}{|\dot{\varepsilon}-1|}>k \varepsilon-T_{r}}{T_{m}-T_{r}}\right]^{\mathrm{m}}\right\} T \leq 400^{\circ} C \\
\sigma_{y}=\left(A+B \varepsilon_{p}^{n}\right)\left(1-C \ln \frac{\dot{\varepsilon}}{\dot{\varepsilon}_{0}}\right)\left\{1-\left[\frac{T+<\frac{\dot{\varepsilon}-1}{|\dot{\varepsilon}-1|}>k \varepsilon-T_{r}}{T_{m}-T_{r}}\right]^{m}\right\} T 400^{\circ} C
\end{array}\right.
$$

$$
\frac{\dot{\varepsilon}-1}{|\dot{\varepsilon}-1|}=\left\{\begin{array}{ll}
1 & \dot{\varepsilon}>1 \\
0 & \dot{\varepsilon} \geq 1
\end{array},\right.
$$

where: $\sigma_{y}$ is plastic stress; $\varepsilon_{p}$ is equivalent plastic strain; $\dot{\varepsilon} / \dot{\varepsilon}_{0}$ is the relative strain rate, in which $\dot{\varepsilon}_{0}$ is strain rate of quasi-static compression test and equals $0.01 \mathrm{~s}-1 ; T$ is the material temperature; $T_{r}$ is ambient temperature; $T_{m}$ is the melting temperature of the material; $A, B, C, n, k$ and $m$ are model parameters, which can be obtained by fitting the experimental data in reference [13], as shown in Table 1.

Parameter values in Johnson-Cook model

Table 1

\begin{tabular}{|c|c|c|c|c|c|c|c|}
\hline Parameters & Temperature & $A / \mathrm{MPa}$ & $B / \mathrm{MPa}$ & $n$ & $C$ & $k$ & $m$ \\
\hline Johnson-Cook & $T \leq 400^{\circ} \mathrm{C}$ & 374 & 795.712 & 0.4545 & 0.01586 & 541.059 & 1.04765 \\
model & $T>400^{\circ} \mathrm{C}$ & 374 & 795.712 & 0.4545 & 0.03293 & 541.059 & 0.30991 \\
\hline
\end{tabular}

\section{Adaptive finite element analysis of bulge formed joint}

According to the adaptive finite element method, the forging process of bulge formed joints is analyzed numerically to study the change of sheet metal thickness in the forming process. The process is as follows:

The initial grid division is as described in Section 3.1, and the grid of plate is as shown in Fig. 5, a, with a total of $12 \times 6 \times 3=216$ elements. The strain energy nephogram is shown in Fig. 6. As can be seen in Fig. 6, the strain energy of some elements is discontinuous, and the stress and equivalent strain of each node can be extracted from the result file. According to the error calculation model, the standard deviation of the nominal strain energy of the two elements in the lower left and right corners of the figure exceeds the given standard $s_{0}=3$, which is consistent with the cloud chart (the standard deviation of the nominal strain energy of some elements is marked in the figure), and the grids need to be further refined.

According to the result, the location of the refined grid is determined. Dichotomy is used to refine the grid, and the result is shown in Fig. 5, a. The calculated strain energy nephogram is shown in Fig. 6. According to the error calculation model, the standard deviation of nominal strain energy error of some nodes is still inaccurate, so the grids need to be refined again, as shown in Fig. 5, b.

The results of the first refinement are shown in Fig. 5, b. A total of 585 elements were generated. After submitting to the software for calculation, the strain energy nephogram obtained is shown in Fig. 7. According to the error calculation model, the stress and equivalent strain of each node are extracted, and the standard deviation of nominal strain energy of each element is calculated. It can be seen from the results that the nominal standard deviation of strain energy of the previously subdivided element has been reduced, but there is still a mesh that needs to be encrypted, as shown in Fig. 5, c.

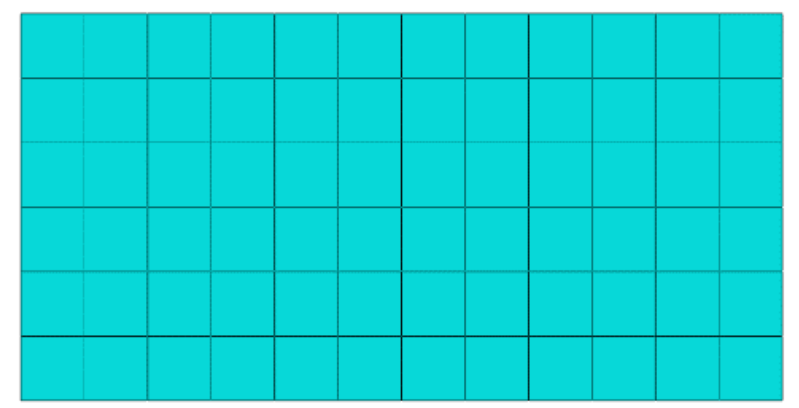

a) The initial grids

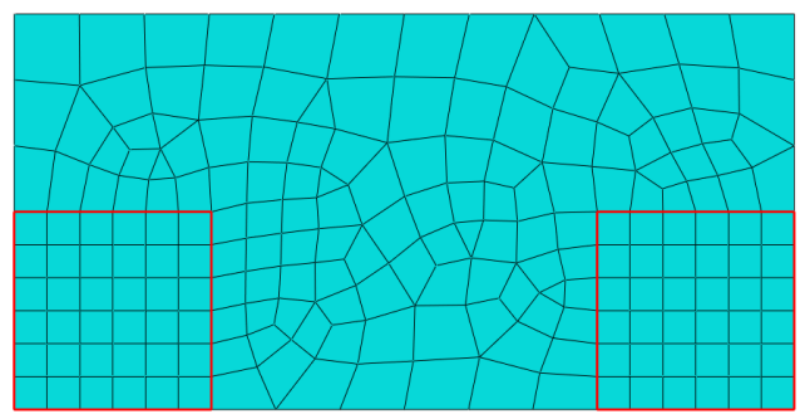

b) The first grids refinement

Fig. 5 The analysis result of initial model 


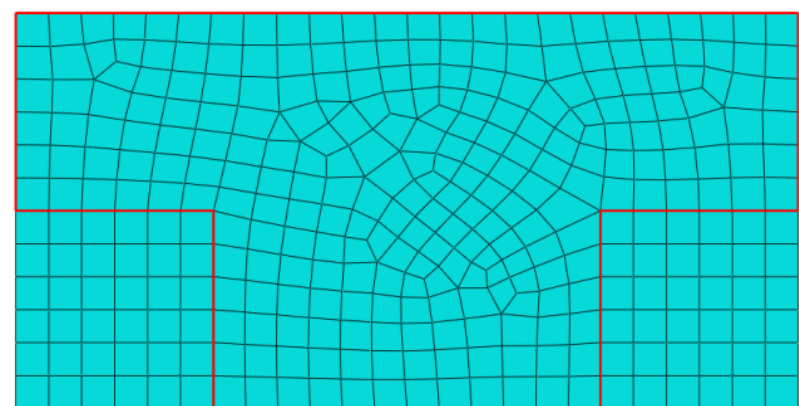

c) The second grids refinement

Fig. 5 Continuion

The second refinement of grids generates 984 elements, as shown in Fig. 5, c. The strain energy nephogram obtained by finite element simulation is shown in Fig. 8. According to the error calculation model, the calculation results have met the accuracy requirements, and the standard deviation of nominal strain energy of each element has met the requirements.

In order to verify the accuracy of the adaptive finite element method proposed in this paper, experiments were carried out to test the thickness changes of different parts of the sheet metal before and after the forming of the bulge formed joint. Fig. 9 shows the layout of measuring points on the bulge formed joint. 101 measuring points are arranged on one node. The grid size is set as the axial spacing of $75 \mathrm{~mm}$ (the spacing at the end part is $50 \mathrm{~mm}$ ), and the circumferential spacing of $103 \mathrm{~mm}$ (about 30 degrees of circumferential angle). The experiment is repeated three times, and Table 2 shows the experimental results of thickness change before and after sheet metal forming.

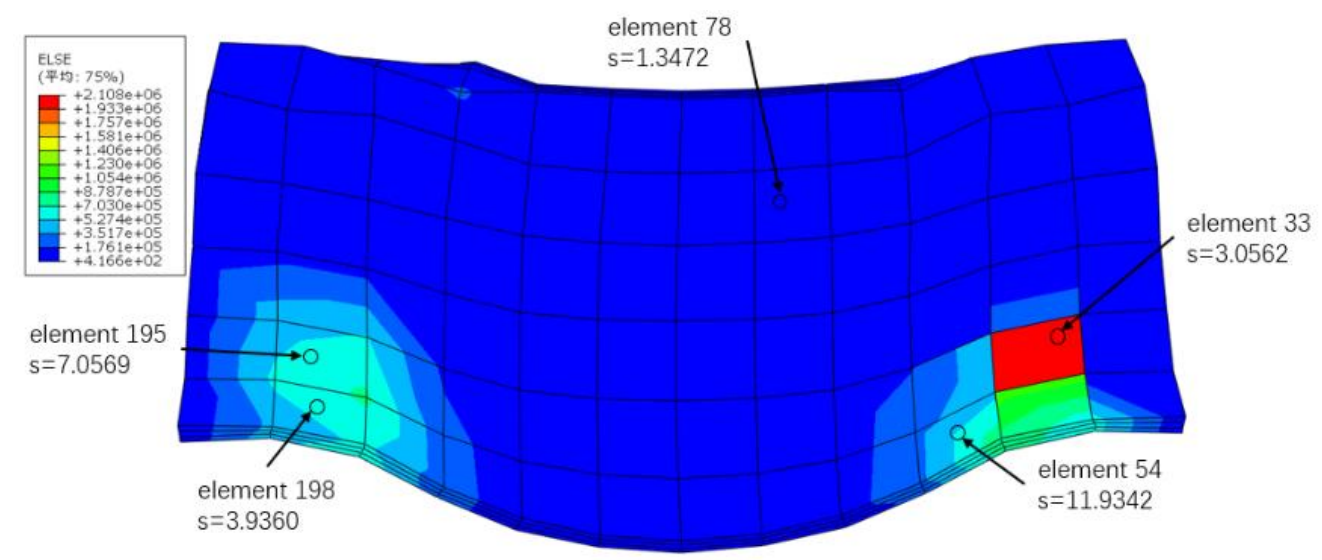

Fig. 6 The analysis result of initial model

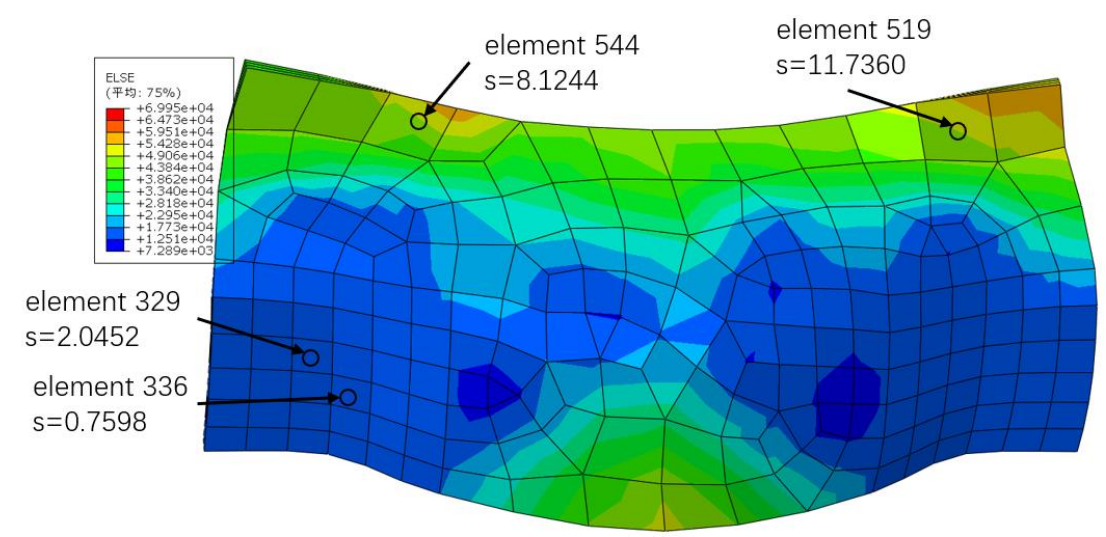

Fig. 7 The analysis result of first grids refinement model

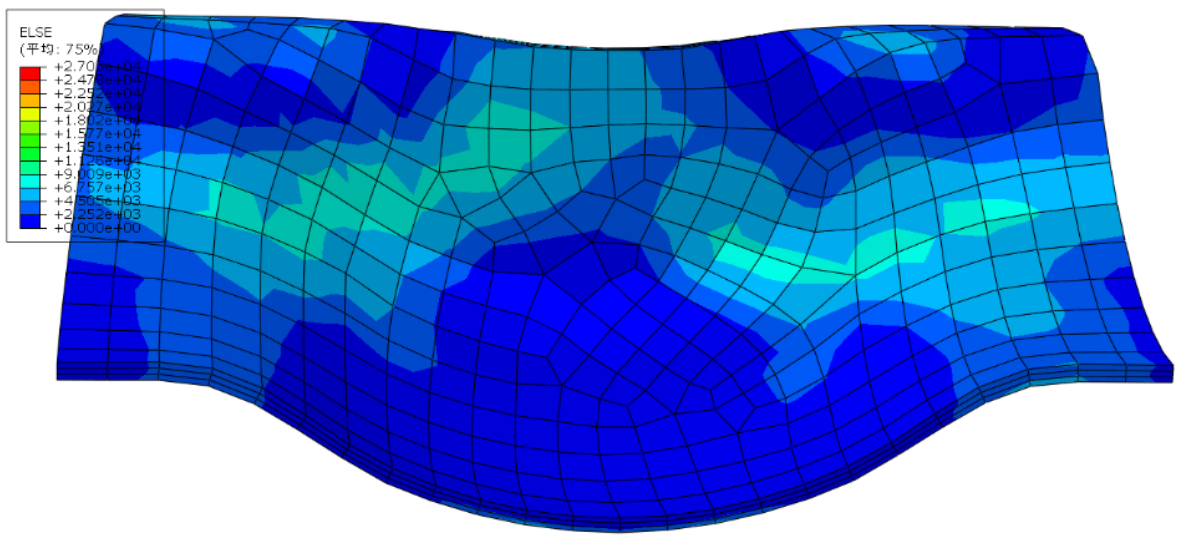

Fig. 8 The analysis result of second grids refinement model 
Table 2 vertices and thinnest points are more accurate than that of

Change of sheet metal thickness in joint forming experiment

\begin{tabular}{|c|c|c|c|c|}
\hline $\begin{array}{c}\text { Position } \\
\text { Cases }\end{array}$ & $\begin{array}{c}\text { Initial thick- } \\
\text { ness, } \mathrm{mm}\end{array}$ & $\begin{array}{c}\text { Vertex, } \\
\mathrm{mm}\end{array}$ & $\begin{array}{c}\text { Thinnest } \\
\text { part, } \mathrm{mm}\end{array}$ & $\begin{array}{c}\text { Thickest } \\
\text { part, } \mathrm{mm}\end{array}$ \\
\hline Case 1 & 14 & 12.4 & 12.2 & 14.7 \\
Case 2 & 14 & 12.5 & 12.5 & 14.1 \\
Case 3 & 14 & 12.5 & 12.3 & 14.3 \\
Average & 14 & 12.4667 & 12.3333 & 14.3667 \\
\hline
\end{tabular}

Table 3 records the comparison between the adaptive finite element simulation results and the experimental results. It can be seen from Table 3 that the initial mesh is relatively rough, and the error of the result is large, the error of the vertex thickness is close to $10 \%$, and the error of the thickest part is as high as $26.27 \%$. However, with refined grids, the accuracy of calculation is improved rapidly. After two times of grids refinement, the error of numerical simulation results reaches an ideal level. The error of the vertex thickness and the thinnest part is within $1 \%$, the error of the thickest part is $3.92 \%$, while the allowable error of engineering is within $5 \%$. At the same time, the simulation results of global refinement (3456 elements in total, equivalent to four times of grid refinement).

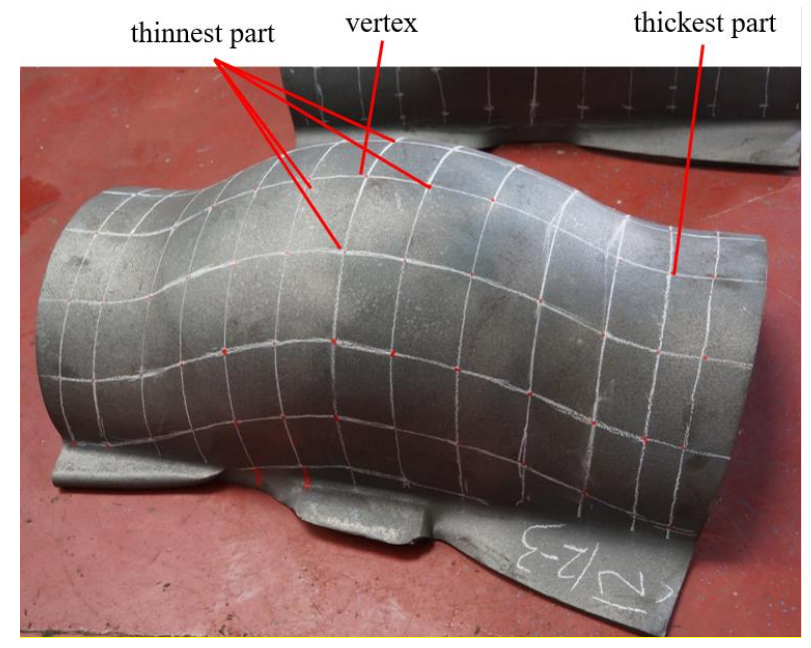

Fig. 9 The layout of measuring points

Comparison of thickness changes in FEM simulation of joint forming with experiment

Table 3

\begin{tabular}{|c|c|c|c|c|c|c|}
\hline & $\begin{array}{c}\text { Thickness of } \\
\text { vertex, mm }\end{array}$ & Error,\% & $\begin{array}{c}\text { Thickness of thin- } \\
\text { nest part, mm }\end{array}$ & Error,\% & $\begin{array}{c}\text { Thickness of thick- } \\
\text { est part, mm }\end{array}$ & Error,\% \\
\hline Initial & 13.7037 & 9.92 & 12.8907 & 4.52 & 17.8042 & 26.27 \\
The first refinement & 13.2858 & 6.57 & 12.3227 & 0.086 & 15.1238 & 7.26 \\
The second refinement & 12.3881 & 0.63 & 12.2925 & 0.33 & 14.6522 & 3.92 \\
Global refinement & 12.1774 & 2.32 & 12.1127 & 1.79 & 14.3154 & 1.52 \\
\hline
\end{tabular}

\section{Conclusions}

Finite element method (FEM) is widely used in numerical simulation, but there are some problems in the face of large deformation and non-linear problems such as plastic forming. In this paper, the forming process of bulge formed joints is analyzed based on adaptive finite element method. The conclusions are as follows:

1 The error calculation model based on the nominal strain energy variance of elements and the grid refinement by dichotomy can be applied to the adaptive finite element analysis method.

2 The results of adaptive finite element simulation and experiment show that the method is feasible. The initial mesh division is rough, the error of calculation results is large, and the error of vertex thickness is $9.92 \%$. With the gradual refinement of the grid, the simulation results are more and more accurate. After the second refinement of the grid, the error between the simulation results and the experimental results is reduced to less than $1 \%$, which shows that the adaptive finite element method proposed in this paper guarantees high accuracy.

\section{Acknowledgement}

This research was supported by the Key Laboratory of Performance Evolution and Control for Engineering Structures (Tongji University), Ministry of Education (No. 2018KF-3), the Science and Technology Committee of Shanghai (No. 19DZ1100202) and the National Natural Science Foundation of China (52075389).

\section{References}

1. Nie, F.; Zhang, Q.; Qin, X.; et al. 2018. Ultimate capacity of bulge formed T-joints under brace axial compressive loading, Thin-Walled Structures 129:237-250. https://doi.org/10.1016/j.tws.2018.02.017.

2. Masoud Marandi, S.; Hassan Nourbakhsh, S.; M. Botshekanan Dehkordi, Hojjat Badnava. 2020. Finite element implementation of coupled temperature-rate dependent fracture using the phase field model, Mechanics of Materials 148:103449.

https://doi.org/10.1016/j.mechmat.2020.103449.

3. Seventekidis, P.; Giagopoulos, D.; Arailopoulos, A.; et al. 2020. Structural health monitoring using deep learning with optimal finite element model generated data, Mechanical Systems and Signal Processing 145 (November-December 2020).

https://doi.org/10.1016/j.ymssp.2020.106972.

4. Xia, F.; Li, H.; Liu, C.; et al. 2019. Forward hot extrusion forming process of 4-lobe aluminum alloy helical surface rotor, Cent. South Univ. 26: 2307-2317. https://doi.org/10.1007/s11771-019-4175-7.

5. Chen, S.; She, C.; Chen, S. 2002. Adaptive elastovisco-plastic finite element analysis of the stability of Dayantang slope, Chinese Journal of Rock Mechanics and Engineering 21(2):169-175 (in Chinese).

6. Li, D. 2009. Research on Adaptive Method Coupling Meshless Method and Finite Element for Distortional Elements of Plates and Shells, D. Shanghai Jiaotong University.

7. Khaji, N.; Zakian, P. 2017. Uncertainty analysis of 
elastostatic problems incorporating a new hybrid stochastic-spectral finite element method, Mechanics of Advanced Materials \& Structures 24(12): 1030-1042. https://doi.org/10.1080/15376494.2016.1202359.

8. Javanmardi, M. R.; Maheri Mahmoud, R. 2019. Extended finite element method and anisotropic damage plasticity for modelling crack propagation in concrete, Finite Elements in Analysis and Design 165:1-20. https://doi.org/10.1016/j.finel.2019.07.004.

9. Sun, Y.; Zhai, J.; Zhang, Q.; et al. 2019. An adaptive meshless method based on friction condition control strategy, Engineering Failure Analysis 106: VSI: ICEFA2018. https://doi.org/10.1016/j.engfailanal.2019.104148.

10. Liu, H.; Cui, T.; Leng, W. 2015. A new hp-adaptive strategy for hp-adaptive finite element methods, Journal on Numerical Methods and Computer Applications 36(2):100-112 (in Chinese).

11. Liu, G.; Tu, Z. 2002. An adaptive procedure based on background cells for meshless methods, Computer Methods in Applied Mechanics \& Engineering 191(17):1923-1943. https://doi.org/10.1016/s0045-7825(01)00360-7.

12. Yu, W.; Zhao, J.; Shi, J. 2011. Dynamic mechanical behaviour of Q345 steel at elevated temperatures: experimental study, Materials at High Temperatures 27(4): 285-293.

https://doi.org/10.3184/096034010X12761931945540.
J. Zhai, Y. Sun, Q. Zhang, X. Qin

FORMING ANALYSIS OF BULGE FORMED JOINTS BASED ON ADAPTIVE FINITE ELEMENT METHOD

\section{S u m m a r y}

Metal bulk forming is widely used because of its own advantages. In order to improve the production efficiency and reduce the cost, numerical simulation is often used to analyze the volume forming process. Because of the large deformation and non-linearity of the forming process, the finite element method (FEM) has the problem of element distortion, which will affect the accuracy and even lead to the failure of the analysis process. In this paper, an adaptive finite element method (AFEM) is proposed to solve this problem. Firstly, the finite element model is established, and grids are roughly divided. After the analysis, according to the error calculation model, the area with large error is determined by the standard deviation of nominal strain energy of nodes. Then, the grids are refined by dichotomy method, and the calculation is continued, repeating this step until the error meets the requirement. Finally, the numerical analysis of the forging process of the bulge formed joint is taken as an example to prove the accuracy of the proposed method.

Keywords: metal forming; adaptive finite element method; error calculation model; refinement strategy; bulge formed joint.

Received September 21, 2019 Accepted April 07, 2021

This article is an Open Access article distributed under the terms and conditions of the Creative Commons Attribution 4.0 (CC BY 4.0) License (http://creativecommons.org/licenses/by/4.0/). 